\title{
Uma solução baseada em internet das coisas para apoiar o turismo de natureza
}

\author{
An internet of things based solution to support nature tourism \\ Una solución basada en Internet de las cosas para apoyar el turismo de naturaleza
}

\section{Resumo}

O setor de turismo apresentou um crescimento sustentável nos últimos anos, observando tanto o setor doméstico quanto o cenário mundial. Embora a pandemia mundial causada pelo COVID-19 tenha interrompido a sequência de crescimento anual do setor e estabeleceu o turismo como o setor mais afetado pela pandemia, estudos apontam que o segundo semestre de 2021 estará relacionado ao início da fase de recuperação do setor e o turismo de natureza é apontado como o nicho mais procurado na fase de recuperação. Por outro lado, o setor de turismo possui um enorme potencial para inovação tecnológica e propostas alinhadas com o Turismo 4.0 podem trazer ainda mais força no processo de busca por destinos turísticos pós-pandemia. Este trabalho propõe uma solução baseada em Internet das Coisas, Computação em Nuvem e Dispositivos Móveis para auxiliar o turismo de natureza. A solução permite que os turistas consigam observar por streaming de vídeo um ponto de visitação remoto à sua localização e avaliar as condições meteorológicas do local em tempo real, permitindo um melhor planejamento da visitação e deslocamento. O estudo contemplou a instalação de 4 (quatro) estações de monitoramento em pontos turísticos do estado de Pernambuco e realizou uma validação inicial da proposta com um pequeno grupo de potenciais usuários da solução. De acordo com os resultados obtidos, as estações de monitoramento fornecem uma informação mais precisa se comparada com serviços existentes e o aplicativo foi bem avaliado pelos potenciais usuários.

Palavras-chave: Turismo de natureza; Tecnologia para o turismo; Turismo 4.0; Internet das coisas; Computação em nuvem; Aplicações para dispositivos móveis.

\footnotetext{
Abstract

The tourism sector has shown sustainable growth in recent years, observing both the Brazilian and the world scenario. Although the global pandemic caused by COVID-19 interrupted the sector's annual growth sequence and established tourism as the sector most affected by the pandemic, studies indicate that the second half of 2021 will be related to the beginning of the sector's recovery phase and tourism of nature is pointed out as the most attractive niche in the recovery phase. On the other hand, the tourism sector has a huge potential for technological innovation and proposals aligned with Tourism 4.0 can bring even more strength in the post-pandemic. This work proposes a solution based on Internet of Things, Cloud Computing and Mobile Devices to help nature tourism. The solution allows tourists to observe via video streaming a remote point of visitation and assess the local weather conditions in real time, allowing for better planning of visitation and displacement. The study included the installation of 4 (four) monitoring stations in tourist spots in the state of Pernambuco and carried out an initial validation of the proposal with a small group of
} 
potential users of the solution. According to the obtained results, the monitoring stations provide more accurate information compared to existing services and the application was well evaluated by potential users.

Keywords: Nature tourism; Tourism technology; Tourism 4.0; Internet of things; Cloud computing; Applications for mobile devices.

\section{Resumen}

El sector turístico ha mostrado un crecimiento sostenible en los últimos años, observando tanto el escenario nacional como el mundial. Si bien la pandemia global provocada por COVID-19 interrumpió la secuencia de crecimiento anual del sector y estableció al turismo como el sector más afectado por la pandemia, los estudios indican que la segunda mitad de 2021 estará relacionada con el inicio de la fase de recuperación del sector y el turismo de naturaleza, se señala como el nicho más buscado en la fase de recuperación. Por otro lado, el sector turístico tiene un enorme potencial de innovación tecnológica y las propuestas alineadas con Turismo 4.0 pueden aportar aún más fuerza en el proceso de búsqueda de destinos turísticos pospandémicos. Este trabajo propone una solución basada en Internet de las Cosas, Cloud Computing y Dispositivos Móviles para ayudar al turismo de naturaleza. La solución permite a los turistas observar a través de la transmisión de video un punto de visita alejado de su ubicación y evaluar las condiciones climáticas locales en tiempo real, lo que permite una mejor planificación de las visitas y el desplazamiento. El estudio incluyó la instalación de 4 (cuatro) estaciones de monitoreo en puntos turísticos del estado de Pernambuco y realizó una validación inicial de la propuesta con un pequeño grupo de potenciales usuarios de la solución. De acuerdo con los resultados obtenidos, las estaciones de monitoreo brindan información más precisa en comparación con los servicios existentes y la aplicación fue bien evaluada por los usuarios potenciales.

Palabras clave: Turismo de naturaleza; Tecnología para el Turismo; Turismo 4.0; Internet de las cosas; Computación en la nube; Aplicaciones para dispositivos móviles.

\section{Introdução}

O setor de turismo apresentou um crescimento sustentável nos últimos anos, observando tanto o setor doméstico quanto o cenário mundial. De acordo com relatório anual de 2019 da World Tourism Organization (UNWTO), o ano de 2018 correspondeu ao $9^{\circ}$ ano consecutivo de crescimento sustentável no setor no mundo (UNWTO, 2019). Contudo, a pandemia mundial causada pelo COVID-19 interrompeu a sequência de crescimento anual do setor e estabeleceu o turismo como o setor mais afetado pela pandemia (Brasil, 2020). Embora os efeitos da pandemia sejam preocupantes, o impacto no setor de turismo precisa ser analisado com base no nicho de turismo analisado e na janela temporal correspondente às fases de: isolamento social; estabilização; e retomada. De acordo com relatório de impacto do COVID-19 produzido pelo governo brasileiro, o segundo semestre de 2021 estará relacionado ao início da fase de recuperação do setor e o turismo de natureza/turismo rural e de aventura está relacionado com o nicho mais procurado na fase de recuperação (Brasil, 2020).

O turismo de natureza é um segmento do turismo que consiste na visitação de territórios predominantemente naturais com o objetivo de apreciar e usufruir da natureza, ou na prática de atividades e experiências diretamente relacionadas com os recursos naturais (Silva, 2013). Embora o turismo de natureza muitas vezes seja associado com regiões que possuem pouca infraestrutura tecnológica, nota-se uma escalada tecnológica no setor turístico, no qual parte significante dos viajantes mundiais recorrem à Internet com frequência para obtenção de informações sobre os seus destinos e outras facilidades (Machado, 2010). Contudo, por mais que essa escalada por suporte tecnológico ao turismo seja perceptível no contexto mundial, há uma carência de estudos e soluções tecnológicas focadas no turismo de natureza e em especial ao contexto brasileiro. Um estudo realizado recentemente conclui que existem poucas soluções tecnológicas para exploração do turismo de natureza no Brasil e as soluções existentes estão associadas a aplicativos que possuem conteúdos voltados para áreas específicas e com grande concentração na região sudeste (Neves, 2020).

Em paralelo a este contexto, há um crescimento mundial no desenvolvimento de soluções inovadoras que utilizem a Internet das Coisas (IoT - Internet of Things) para proporcionar agilidade, acurácia e eficiência na tomada de decisões mais assertivas em diversos setores, como agronegócio, Industria 4.0, Cidades Inteligentes, entre outros (Choudhari, 2017). Porém, no setor turístico brasileiro, segundo Santa Ana (Santana, 2019) as soluções digitais ou tecnológicas existentes ainda estão focadas em tecnologias incipientes como a Web 2.0 ou aplicativos móveis sem inteligência, o que demonstra a necessidade de 
desenvolvimento de soluções inovadoras que resultem em um Turismo 4.0 (Stankov, 2020), por meio de tecnologias diruptivas como: Inteligência Artificial, Internet das Coisas, Big Data, Computação em Nuvem, Robótica, dentre outras. Essas novas tecnologias possuem potencial para mudar completamente a experiência dos turistas com soluções personalizadas, rapidez nas respostas, conveniência e agilidade.

A proposta deste trabalho é fornecer uma Prova de Conceito $(\mathrm{PdC})$ de uma solução tecnológica para apoiar o Turismo 4.0, com foco no nicho de turismo de natureza. A ideia é que o turista consiga avaliar as condições de um ponto turístico remoto antes de se locomover até o destino. Um exemplo típico está relacionado com a prática de esportes ao ar livre que exijam condições específicas de clima e tempo como voo livre, windsurf, trilhas ecológicas, e assim por diante. A solução proposta é baseada em IoT e integra equipamentos, como estações meteorológicas, câmeras de vídeo e controladores, computação em nuvens, entre outros, oferecendo os serviços ao turista por meio de um aplicativo para dispositivos móveis. Este artigo corresponde à descrição da solução proposta, tanto por meio do detalhamento técnico da arquitetura usada para as estações de monitoramento, quanto dos detalhes do aplicativo desenvolvido. Além disso, o artigo fornece resultados da PdC em termos de testes de usabilidade com grupos de pessoas que usaram o aplicativo com estações posicionadas em pontos turísticos do estado de Pernambuco.

\subsection{Revisão da literatura}

Diversos esforços foram concentrados nos últimos anos com relação ao uso de tecnologia voltada para o Turismo e este movimento é percebido tanto no contexto nacional quanto internacional. Khatri conduziu um estudo que compilou 10 anos de publicações sobre o emprego de Tecnologia da Informação (TI) voltada para o setor de Turismo (Khatri, 2019). De acordo com este levantamento, a TI no setor de turismo é mais comumente usada para atender às necessidades básicas de informação, estudo de comportamento e desempenho, e gerenciamento do processo de operação. Outras tarefas em potencial como promoção, marketing, processos de gerenciamento de clientes e criação de valor/vantagem competitiva ainda correspondem a uma lacuna na área e com potencial de exploração futura.

Buhalis analisa o uso atual de Tecnologia da Informação e Comunicação (TIC) no turismo, além de fornecer prognósticos sobre o direcionamento futuro da área para um ambiente de inteligência no Turismo (smart tourism) (Buhalis, 2019). O estudo elenca os marcos tecnológicos mais importantes nos últimos anos, incluindo desde facilidades gerais da Web que viabilizaram eTurismo (como a revolução causada pelos motores de busca como Google e Yahoo e o surgimento de sites especializados no setor de viagens e turismo); passando por soluções mais específicas como o TripAdvisor, que permite que os clientes possam expressar online a sua opinião sobre experiências de viagens, com o foco na geração de conteúdo pelo próprio turista (Buhalis, 2019). Contudo, o estudo também procura diferenciar a mera aplicação de TIC para sistematizar informações úteis do Turismo de um novo cenário baseado em um "Turismo Inteligente" a partir de 2020, que é caracterizado pela presença de diversas tecnologias disruptivas, tais como: a Internet das Coisas; redes móveis de quinta geração (5G); identificação por radiofrequência (RFID); smartphones e dispositivos vestíveis; impressão 3D; criptomoeda e blockchain; computação pervasiva; gamificação; bem como recursos analíticos aprimorados com suporte de Inteligência Artificial (IA) e aprendizagem de máquina. Todas essas tecnologias criam a infraestrutura e a rede digital inteligente que viabilizam a interoperabilidade perfeita entre todos os envolvidos. E essa interconexão inteligente promove fluidez entre as interações físicas e digitais, capacitando redes dinâmicas em constante evolução e impondo uma mudança disruptiva na indústria de turismo como é conhecida atualmente (Buhalis, 2019).

Por outro lado, uma avaliação geral do cenário brasileiro com relação ao uso de TIC para o Turismo sugere que a maior parte das soluções tecnológicas nacionais para o setor também estão concentradas no conceito já estabelecido de eTurismo baseado na Web e em sistematização de informações para apoiar o setor. A carência de soluções inteligentes é ainda 
mais percebida quando a análise é especializada para o turismo de natureza. Uma pesquisa exploratória recente realizada por Neves e colaboradores demonstrou que existem em torno de 14 aplicativos voltados para exploração do turismo de natureza no Brasil e que atendem parcialmente às demandas do segmento (Neves, 2020). O estudo apontou que os aplicativos na maior parte das vezes possuem conteúdo específico para um determinado ponto turístico e a maior quantidade está concentrada na região sudeste, de modo que parques de regiões com maior potencialidade turísticas não dispõe de inserção tecnológica. O estudo conclui que há pouca usabilidade de recursos de tecnologia móvel no turismo de natureza (Neves, 2020). Contudo, já existem iniciativas na academia relacionadas com a aplicação das tecnologias disruptivas citadas por (Buhalis, 2019) para apoiar a próxima geração de soluções para viabilizar o smart tourism.

Gcaba e Dlodlo fazem um levantamento do turismo na África do Sul e fornecem ideias de soluções baseadas em IoT para o turismo do país, com destaque para a definição da arquitetura de um sistema baseado em IoT para identificação automática de incêndio florestal (Gcaba \& Dlodlo, 2016). Garcia e colaboradores exploram o conceito de gamificação em dispositivos móveis como tecnologia habilitadora para o turismo inteligente (GARCIA et al, 2018). Pasanen e colaboradores focam em soluções para o turismo de natureza baseadas em realidade virtual, comparando o uso de tablets e de óculos especiais para realidade virtual (Pasanen, et al., 2019). Neste contexto, este estudo também procura avançar na proposição de soluções inteligentes para apoiar o Turismo baseadas em tecnologias disruptivas, integrando dispositivos IoT, computação em nuvem e computação móvel para exploração do turismo de natureza.

\section{Metodologia}

O desenvolvimento deste projeto considerou diversas macro-etapas, incluindo desde uma fase de pesquisa exploratória relacionada com uma revisão da literatura detalhada (Pereira, et al., 2018) até uma fase mais intervencionista, na qual um protótipo da solução proposta foi disponibilizado em diferentes regiões do estado de Pernambuco para permitir que o público-alvo da solução pudesse opinar sobre a proposta. Além disso, em algumas das etapas foi aplicada uma abordagem quantitativa (como na análise de desempenho da infraestrutura em nuvem), mas a maior parte dos estudos foram conduzidos de forma qualitativa (como no caso dos testes de usabilidade). De forma geral, a metodologia empregada neste projeto pode ser resumida pelas macro-etapas ilustradas na Figura 1.

A Revisão da Literatura considerou uma pesquisa exploratória nos principais motores de busca para publicações científicas no período de 2010 até 2020 e fazendo uso das seguintes palavras chaves: Turismo de Natureza; Tecnologia para o Turismo; Turismo 4.0; Internet das Coisas; Computação em Nuvem; Aplicações para Dispositivos Móveis; Nature Tourism; Tourism Technology; Tourism 4.0; Internet of Things; Cloud Computing; e Applications for Mobile Devices. Após a busca inicial, as principais publicações foram selecionadas considerando a relevância dos veículos, leitura parcial dos artigos e leitura completa de um subgrupo selecionado. A revisão da literatura atuou como um ponto de partida para a etapa de Concepção da Arquitetura Geral da Proposta. Esta segunda etapa foi responsável pela especificação de uma macro-solução composta por alguns dispositivos IoT para monitoramento e por uma ideia geral de aplicativo que pudesse exibir os dados monitorados, mas sem o detalhamento necessário para evolução do protótipo, o que ocorreu nas etapas 3 e 4 da Figura 1. Na etapa 3 foram detalhadas 3 (três) soluções técnicas alternativas para representar a arquitetura das estações de monitoramento e estas variações de soluções alternativas consideraram diferentes compromissos entre qualidade dos equipamentos, custo financeiro, facilidade de fornecedores para os dispositivos individuais e assim por diante. A etapa 4 corresponde a um processo de desenvolvimento de software típico, voltado para a viabilização de um aplicativo Android que pudesse exibir os dados coletados pelas estações de monitoramento, incluindo: levantamento de requisitos, ideação das telas do sistema, protótipo de alta fidelidade, detalhamento de requisitos, detalhamento técnico orientado a objetos, implementação de código-fonte em linguagem Java, testes e estabilização do código-fonte, testes de usabilidade. Finalizadas as etapas de definição detalhada da arquitetura das 
estações e desenvolvimento do aplicativo Android, os equipamentos necessários foram adquiridos e a solução completa foi avaliada pela equipe interna, em ambiente controlado. Em seguida, a etapa 6 considerou um outro nível de avaliação da solução completa, desta vez em ambiente externo. Nesta fase, as estações de monitoramento foram instaladas em 4 (quatro) diferentes pontos turísticos do estado de Pernambuco, correspondendo a diferentes variações climáticas e variações de paisagens. Após a implantação da solução em ambiente externo, a equipe do projeto monitorou as estações, avaliando questões diversas como resposta às intempéries, nível de conectividade (uso de rede e Internet), disponibilidade e avaliação básica de desempenho. Finalmente, a solução proposta foi divulgada para o público em geral para que uma avaliação externa e mais ampla pudesse ser considerada.

Figura 1 - Ilustração da metodologia empregada no projeto, considerando 8 (oito) macro-etapas.

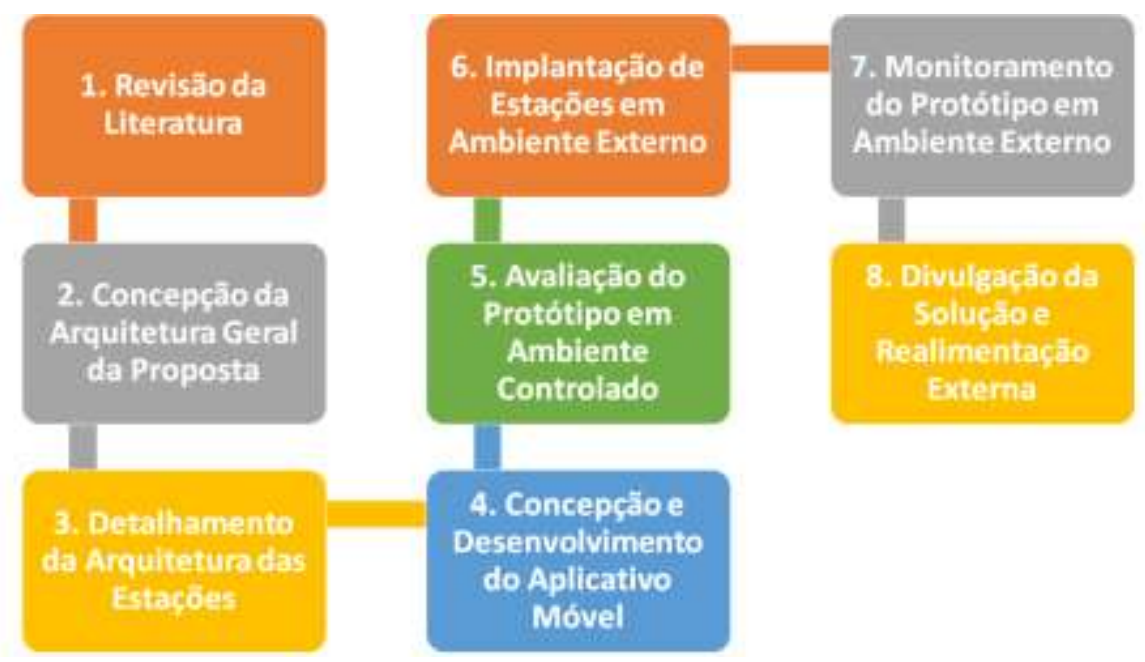

Fonte: Autores.

\section{Detalhamento da Proposta}

A arquitetura geral da solução proposta é formada por uma estação de monitoramento, uma infraestrutura de comunicação, servidores em nuvem e aplicativo para dispositivos móveis, como ilustra a Figura 2. As estações de monitoramento, localizadas nos pontos turísticos inteligentes, são compostas por: câmeras de vídeo, estações meteorológicas e demais componentes de infraestrutura para controle e comunicação (controladores equipados com interfaces de rede). Nesta arquitetura, as estações de monitoramento levarão os dados coletados para um servidor da aplicação hospedado na nuvem e estes dados serão consumidos pelos turistas por meio de um aplicativo para dispositivos móveis. Nesta fase de PdC, a plataforma-alvo para o aplicativo foi Android, mas uma versão compatível com outras plataformas poderá ser disponibilizada no futuro. Com base no entendimento geral da solução, é possível descrever os detalhes técnicos das estações de monitoramento (Seção 3.1) e do aplicativo proposto (Seção 3.2). 
Figura 2 - Visão de alto nível da solução proposta.

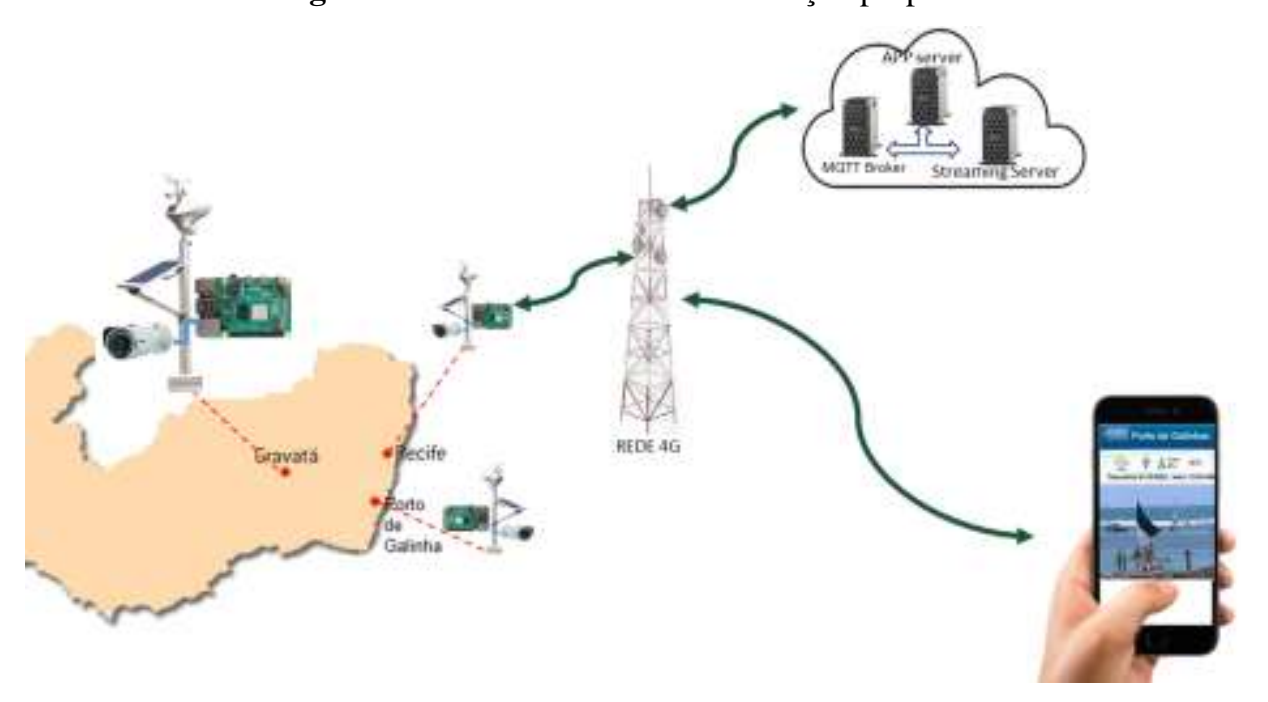

Fonte: Autores (2021).

\subsection{Detalhamento das estações de monitoramento}

Para atender aos requisitos do projeto, a concepção das estações de monitoramento baseou-se na integração de quatro dispositivos, sendo três dispositivos de Hardware compostos por uma estação meteorológica com dez sensores, uma câmera de alta definição e um minicomputador de placa única baseado na arquitetura Caninos Labrador ${ }^{1}$, integrados a um quarto dispositivo virtual, denominado servidor em nuvem, provido com sistema operacional Linux Ubuntu, embarcado com a plataforma Dojot de Internet das Coisas (CPqD, 2017) e plataforma de distribuição de Streaming $N G I N X^{2}$. A Figura 3 ilustra a topologia de uma estação de monitoramento em detalhes, e seus componentes são apresentados individualmente nas subseções a seguir.

\subsubsection{Estação meteorológica}

A estação meteorológica empregada no projeto foi projetada e construída pela empresa nacional B2K Tech, sendo uma solução de monitoramento climático on-line de fácil implementação, visto que conta com um painel solar e bateria interna, não necessitando de utilização de energia elétrica, sendo necessário apenas a fixação da estação no solo e conexão de rede (B2K Tech, 2020). A transmissão dos dados meteorológicos coletados é realizada em tempo real através de rede sem fio de alta velocidade ou por rede móvel celular à plataforma em Nuvem. A estação conta com 10 (dez) sensores para monitoramento contínuo, sendo eles: temperatura; umidade; precipitação; direção do vento; velocidade do vento; rajada de vento; intensidade luminosa; incidência de raios ultravioleta; pressão atmosférica e localização (GPS). Vale a pena ressaltar que a primeira versão projetada do aplicativo para dispositivos móveis considerou uma subseleção dos dados provenientes destes 10 sensores de modo a fornecer uma interface gráfica mais limpa e com informações consideradas essenciais para o turismo de natureza.

\footnotetext{
${ }^{1} \mathrm{https}: / /$ caninosloucos.org/pt/

${ }^{2}$ https://nginx.org/en/
} 
Figura 3 - Topologia de uma estação de monitoramento.

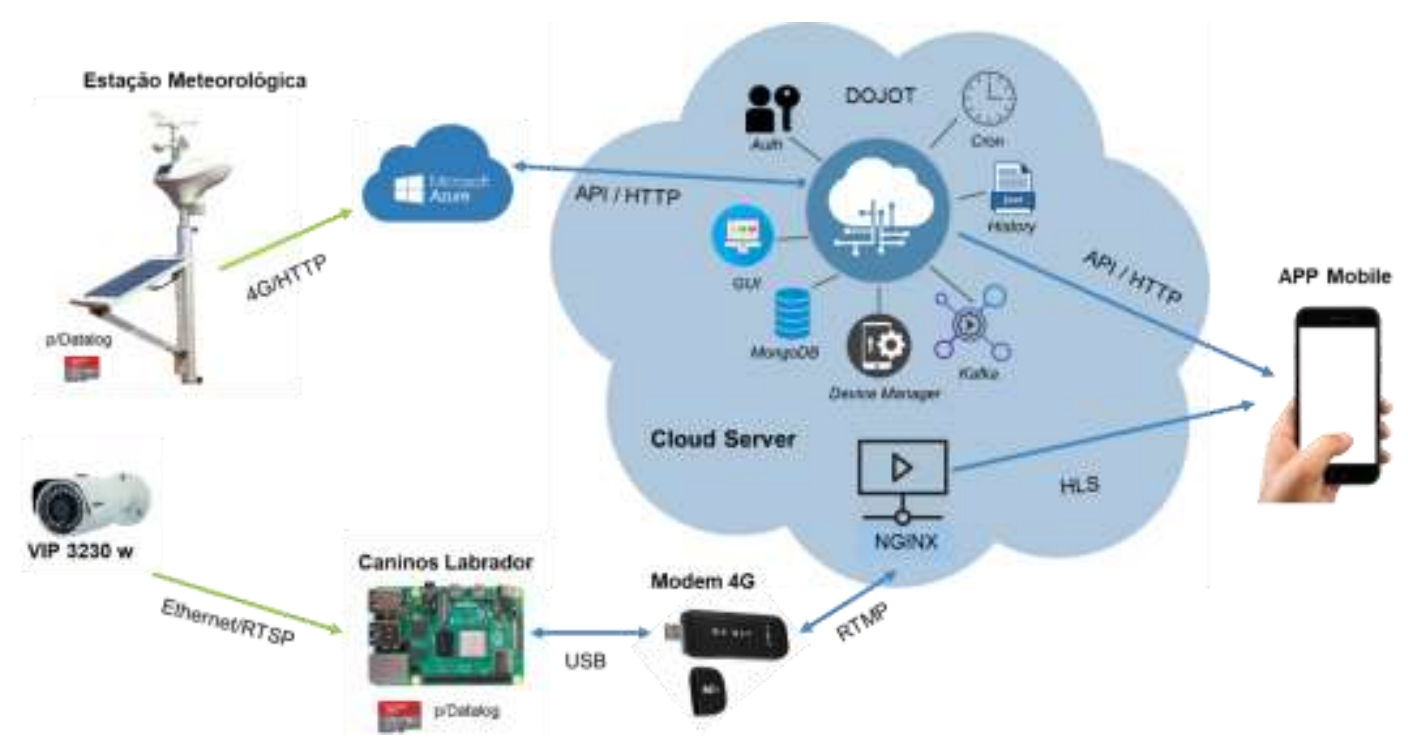

Fonte: Autores.

\subsubsection{Câmera IP}

Para proporcionar aos usuários uma experiência mais imersiva dos pontos turísticos, buscou-se implementar câmeras de vídeo nas estações de monitoramento, onde optou-se pela utilização de câmeras IP de alta definição, fabricadas pela Intelbras, indústria nacional de soluções de segurança e redes. A escolha deste tipo de câmera justificou-se por serem capazes de transmitir imagens através de redes sem fio, por suportarem modernos protocolos de compressão, por apresentarem fácil configuração e gerenciamento, além de serem de fácil integração com a solução de streaming proposta para o projeto. Utilizouse o protocolo RTSP, Real Time Streaming Protocol (Schulzrinne, 1998) para integrar a câmera IP ao computador de placa única Caninos Labrador (Caninos, 2018), devido a este protocolo possuir recurso de controle de transferência em tempo real, evitando a perda de pacotes, consequentemente garantindo a qualidade da transmissão de vídeo.

\subsubsection{Computador de placa única Caninos Labrador}

Para que fosse possível realizar a transmissão de vídeo da câmera integrada à estação de monitoramento até o aplicativo móvel, adotou-se o uso de minicomputadores de placa única para Internet das Coisas (IoT) de modelo Labrador, desenvolvidos pelo grupo de trabalho Caninos, uma iniciativa da Instituição Tecnológica LSI-TEC (Laboratório de Sistemas Integráveis Tecnológico) em parceria com a escola politécnica da Universidade de São Paulo (USP). A placa Caninos Labrador utiliza um processador de arquitetura $A R M v 8$ de 64 bits, conta com 2GB de memória $R A M$ e 16GB de memória interna, suporta codificação de vídeos em qualidade Full HD com codec H264, além de ser embarcado com sistema operacional Linux Debian 10 (Caninos, 2018).

A placa Labrador foi posicionada como um gateway e sua integração com a câmera IP foi implementada através de rede sem fio e protocolo de transmissão RTSP, onde adotou-se o software $F F M P E G^{3}$ para realizar o encapsulamento do vídeo com protocolo RTMP e transmissão para o servidor em nuvem com protocolo HTTP. Para gerenciamento remoto da placa Labrador, utilizou-se uma solução de túnel de rede com protocolo criptografado SSH.

\footnotetext{
${ }^{3}$ http://ffmpeg.org/
} 


\subsubsection{Ambiente computacional em nuvem}

A gestão dos recursos de internet das coisas (IoT) foi centralizada em um ambiente computacional em nuvem, para tanto, implementou-se a solução Cloud Computing fornecida por UOL HOST, que consiste em um servidor Linux Ubuntu LTS, com processador de quatro núcleos virtuais, dispondo de 4 GB de memória $R A M$ e 50 GB de armazenamento, além de contar com $50 \mathrm{MB} / \mathrm{s}$ de largura de banda e disponibilizar um IP público. A utilização de uma plataforma de IoT em nuvem permite criar um ecossistema de dispositivos integrados, facilitando a comunicação entre sistemas e proporcionando o gerenciamento de comunicação, fluxo e coleta de dados para análise e execução de ações (Embarcados, 2019). Diante disto, optou-se por utilizar no projeto a plataforma Dojot (CPqD, 2017), uma plataforma de Internet das Coisas gratuita, desenvolvida no Brasil pelo Centro de Pesquisa e Desenvolvimento em Telecomunicações (CPqD). Seus principais recursos incluem: APIs abertas para fácil acesso aos recursos da plataforma; conexão e coleta de dados de dispositivos; processamento de eventos em tempo real; plataforma multisserviço para execução simultânea de diversos protocolos de comunicação; interface gráfica em ambiente Web para configuração de dispositivos; APIs de autenticação, de controle de fluxo de processamento de dados e de controle de eventos nos bancos de dados. Com a implementação da Dojot no projeto foi possível coletar os dados climáticos fornecidos pelas estações meteorológicas através de requisições HTTP em Rest API, consolidar as informações em um banco de dados e disponibilizá-los para a aplicação móvel em formato JSON. Para que fosse possível se atingir um alto nível de escalabilidade na distribuição de streaming das câmeras, implementou-se uma solução NGINX, um servidor web de código aberto que pode ser utilizado como servidor HTTP, proxy reverso, proxy de e-mail, balanceador de carga ou servidor de mídia (EVEO, 2021). O serviço foi implementado como servidor de mídia no ambiente computacional em nuvem do projeto, aproveitando-se de sua alta capacidade de lidar com múltiplos streamings em alta definição para trazer aos usuários do aplicativo Smart Tour PE uma experiência de qualidade de serviço (QoE - Quality of Experience) durante a visualização das câmeras dos pontos turísticos.

\subsection{Detalhamento do aplicativo para dispositivos móveis}

Foi desenvolvido um aplicativo para sistema operacional Android denominado Smart Tour PE, compatível com versões superiores a 6.0 (Marshmallow). O Smart Tour PE é um aplicativo completo de apoio aos turistas, que se utiliza de dispositivos de IoT para prover informações climáticas e visuais às pessoas que desejam visitar ou se informar acerca das atrações cadastradas. Estações climáticas e câmeras de monitoramento transmitem streaming de vídeo e informações climáticas em tempo real para a nuvem, e através do aplicativo o turista consegue visualizar estas informações em seu dispositivo móvel, pautando seu roteiro de acordo com as peculiaridades de cada local que deseja visitar. Recursos como geolocalização, chat, informações pessoais, botão de pânico, e informações gerais de apoio ao turista também foram idealizadas para o aplicativo, porém não foram implementadas na versão inicial, que conta apenas com uma implementação básica das principais funcionalidades. O usuário ao acessar o aplicativo deve selecionar o idioma de sua preferência e cadastrar-se para ter acesso às demais funcionalidades. Assim que ele tem acesso ao aplicativo, consegue visualizar cards com imagens e informações sobre os pontos turísticos atualmente cobertos pela aplicação. Ao selecionar um card, ele tem acesso às informações climáticas e ao streaming de vídeo pertinente ao ponto selecionado. Também está disponível a tela de dúvidas frequentes (FAQ), na qual o usuário pode sanar dúvidas sobre as funcionalidades atualmente disponíveis.

Os requisitos funcionais inicialmente listados para o aplicativo foram: RF01: Permitir ao usuário registrar-se no aplicativo; RF02: Permitir ao usuário efetuar login no aplicativo; RF03: Permitir ao usuário recuperar sua senha; RF04: Permitir ao usuário consultar pontos turísticos para visitação; RF05: Permitir ao usuário consultar as condições climáticas dos pontos turísticos disponíveis; RF06: Permitir ao usuário visualizar streaming de vídeo e fotos dos pontos turísticos; RF07: Permitir ao usuário consultar um mapa para orientação; RF08: Permitir ao usuário utilizar geolocalização para localizar sua 
posição em um mapa; RF09: Permitir ao usuário consultar informações detalhadas sobre eventos que estejam ocorrendo nos pontos turísticos; RF10: Permitir ao usuário consultar informações sobre autoridades locais como endereços eletrônicos e telefones de apoio em caso de emergência; RF11: Permitir ao usuário acessar um chat para contactar autoridades locais; RF12: Permitir ao usuário a utilização de um botão de pânico para uso em situações de iminente emergência; RF13: Permitir ao usuário consultar um relatório estatístico sobre suas atividades no aplicativo.

Os requisitos não-funcionais elencados inicialmente para o projeto foram: RNF 01: Permitir o envio de notificações agendadas pelo aplicativo; RNF 02: Permitir ao usuário avaliar o local visitado; RNF 03: Permitir ao usuário alterar informações de seu perfil; RNF 04: Permitir ao aplicativo a coleta de dados para fins estatísticos e pesquisa; RNF 05: O aplicativo deve ser capaz de acessar dados disponíveis na nuvem; RNF 06: O aplicativo deve ser capaz de executar em aparelhos compatíveis com sistema Android 6.0; RNF 07: A interface gráfica do aplicativo deve ser intuitiva e de fácil manuseio para o usuário; RNF 08: O sistema de mapas deve ser acessível a no máximo 3 clicks a partir de qualquer tela do aplicativo.

Figura 4 - Diagrama UML do App Smart Tour PE.

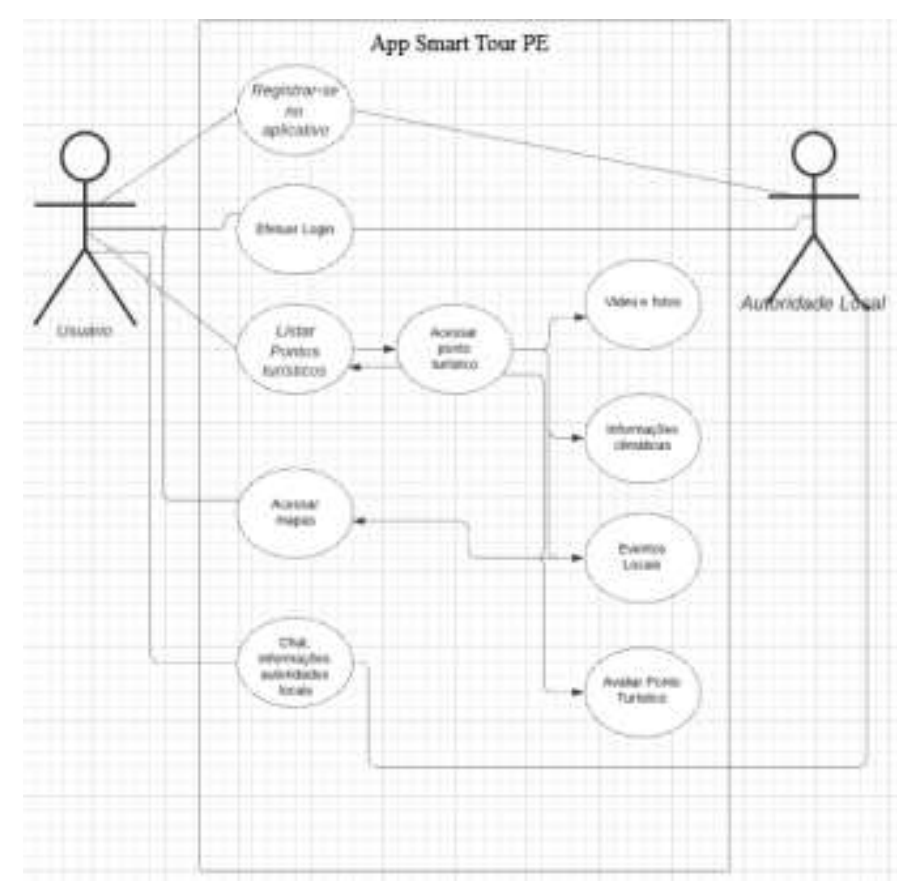

Fonte: Autores.

A Figura 4 apresenta o diagrama UML do aplicativo, contendo os principais meios de interação entre os atores do sistema, bem como o fluxo lógico de telas. A Figura 5 demonstra um mapeamento básico do banco de dados idealizado para o aplicativo, por meio de um DER (Diagrama de Entidade e Relacionamento).

\subsubsection{Tecnologia de desenvolvimento}

Por questões de aproveitamento de conhecimento tácito da equipe e de mitigação de riscos de projeto, o desenvolvimento do aplicativo foi realizado em Android nativo com a linguagem JAVA (Flauzino, 2018). Os dados de usuário são armazenados em um banco de dados local Sqlite (SQLite, 2021). Os dados utilizados pelo aplicativo provêm de um banco de dados remoto, implantado no backend e consultado a partir de requisições HTTP (HyperText Transfer Protocol). Essa arquitetura de consulta a banco remoto e persistência em banco local foi escolhida por garantir uma estabilidade maior da 
aplicação já que mesmo que o usuário esteja sem sinal de Internet, poderá consultar a última persistência de dados em banco local. A consulta ao banco remoto é feita sempre que o usuário acessa o aplicativo e caso alguma mudança tenha sido feita no backend (como a inclusão de novos pontos turísticos), a aplicação irá receber o sinal e fará a sincronização dos dados.

Figura 5 - Diagrama de entidade relacionamento do app Smart Tour PE. Fonte: os autores.

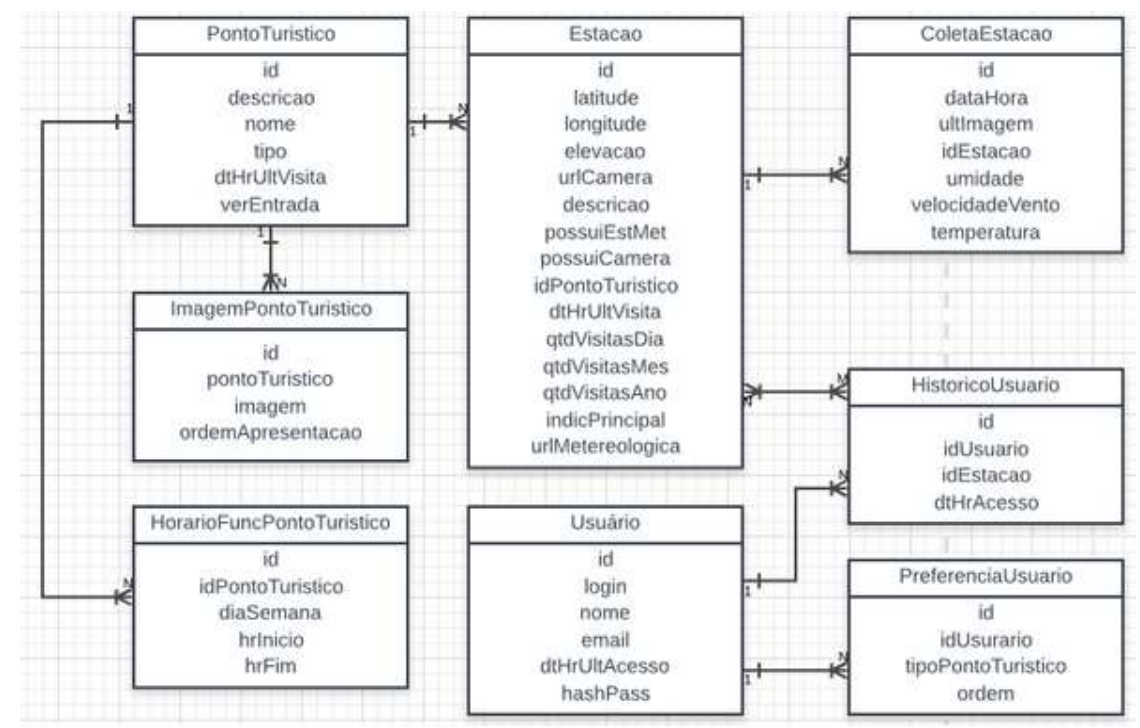

Fonte: Autores.

A tela principal foi implementada com um RecyclerView utilizando CardView, e, a implementação seguiu a recomendação da Google (Developer, 2021). Os cards são interativos com a experiência do usuário e sinalizam quando o ponto turístico, ao qual representam, é um ponto turístico relativo a uma praia ou uma cidade, se possui câmera de transmissão de streaming de vídeo em tempo real e se o usuário já fez uma visita virtual ao ponto turístico, entre outras sinalizações. A Figura 6 ilustra a tela principal e a tela de detalhes que surge após o usuário clicar no card. Neste exemplo em específico, o ponto turístico exibido corresponde à praia de Porto de Galinhas, localizada no município de Ipojuca, em Pernambuco. A partir do momento em que o usuário clica em um dos cards ele estará fazendo uma visita virtual ao ponto turístico e uma tela de detalhes sobre o ponto irá ser exibida. Os dados recuperados no banco, no momento de transição de telas entre a tela principal e a tela de detalhes, estão mantidos em listas e são passados de uma tela para outra utilizando. Na tela de detalhes há uma foto do ponto turístico e um texto com maiores informações sobre o ponto visitado, além de informações climáticas em tempo real. As informações climáticas são obtidas de forma assíncrona, com AsyncTask, e atualizadas enquanto o usuário observa a tela de detalhes (Developer, 2021). Se o ponto turístico possuir uma câmera de vídeo instalada, a foto contará com um botão de play em sua composição e o usuário poderá assistir a um streaming de vídeo do ponto turístico. Para a exibição do streaming de vídeo, foi implementado um $V L C$ embarcado na aplicação (Developer, 2021), o que habilita a aplicação a tanto executar streamings enviados por protocolos RTSP quanto ao protocolo HLS. 
Figura 6 - Tela principal e tela de detalhes do aplicativo.

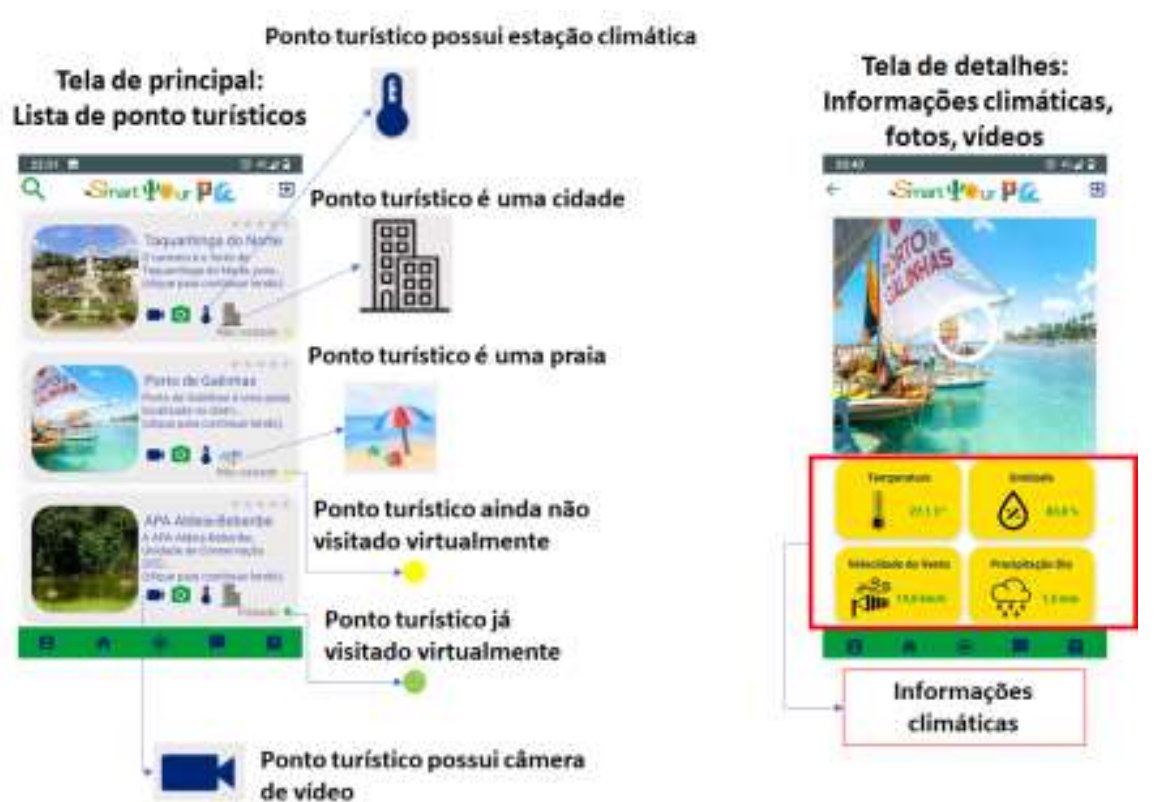

Fonte: Autores.

\section{Resultados e discussão}

\subsection{Avaliação geral dos pontos monitorados}

Para efeito de PDC, foram instaladas 4 estações de monitoramento em diferentes municípios do estado de Pernambuco, conforme ilustrado na Figura 7, a saber: estação (A) no município de Taquaritinga do Norte; estação (B) no município de Gravatá; estação (C) no município de Camaragibe; e estação (D) instalada no município de Ipojuca. As localidades do estudo foram escolhidas levando em consideração a vocação turística dos pontos, questões de segurança física, conectividade e de modo a ilustrar diferentes climas e paisagens. O município de Taquaritinga do Norte possui o segundo ponto mais elevado do estado de Pernambuco, com altitude em torno de $1200 \mathrm{~m}$, apresentando um temperaturas amenas na maior parte do ano (média anual em $18^{\circ} \mathrm{C}$ ). Taquaritinga do Norte é procurada por turistas que desejam apreciar o clima de serra e praticar atividades esportivas diferenciadas como o voo livre. O município de Gravatá fica localizado em outra região elevada do estado de Pernambuco (o planalto da Borborema). Gravatá é conhecido por oferecer programas turísticos relacionados com as atividades do campo e pela culinária típica da região. O ponto (C) corresponde à Área de Preservação Ambiental (APA) Aldeia-Beberibe, que se estende por vários municípios de Pernambuco e que neste projeto foi representada por um ponto localizado no município de Camaragibe. A APA Aldeia-Beberibe oferece diversas oportunidades para exploração das riquezas da Mata Atlântica, como trilhas ecológicas, passeios de cavalo, prática de mountain bike, etc. O ponto (D) corresponde à praia de Porto de Galinhas, no município de Ipojuca. Porto de Galinhas foi eleita 10 vezes consecutivas como a melhor praia do Brasil por uma revista especializada do setor de Turismo. Porto de Galinhas possui temperaturas altas, típicas do clima tropical, na maior parte do ano. É conhecida pela beleza das piscinas naturais de águas cristalinas e pela prática de atividades esportivas aquáticas diversas. As estações foram instaladas na maior parte dos casos em pontos elevados, de modo a permitir uma aferição precisa dos dados meteorológicos e obter uma visão panorâmica do local. A Figura 8 ilustra as estações instaladas nos locais indicados pela Figura 7. No caso da estação (C), foi necessária a instalação diretamente no solo e por este motivo uma grade de proteção foi acrescentada para evitar o acesso de pessoas não autorizadas (Figura 8c). 
Figura 7 - Ilustração da localização das estações de monitoramento instaladas.

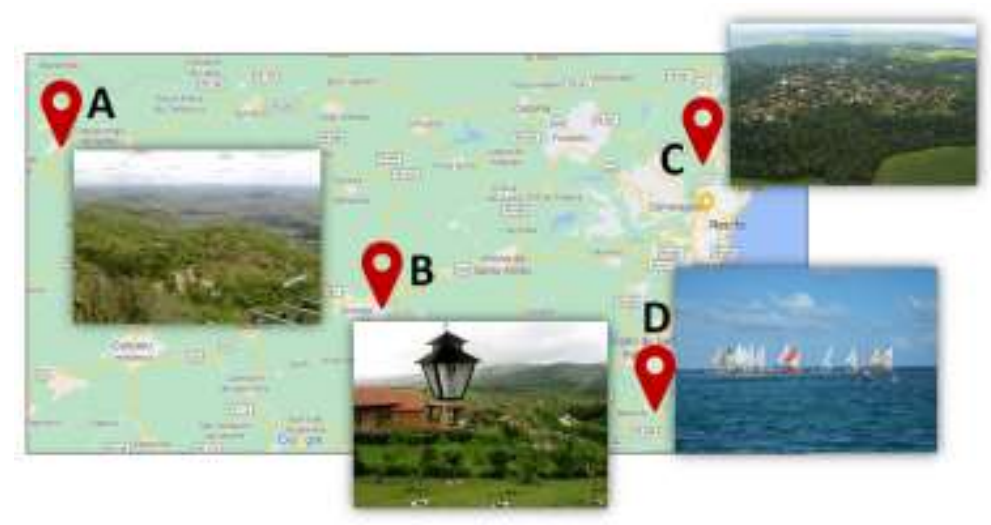

Fonte: Autores.

Figura 8 - Ilustração das estações instaladas nos pontos indicados na Figura 7.

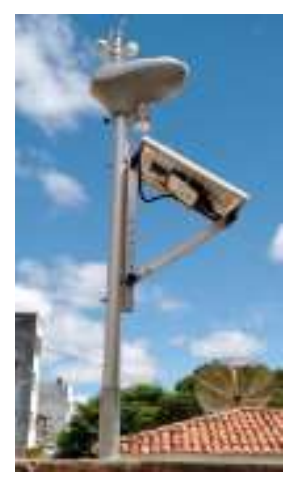

(a) Taquaritinga do Norte

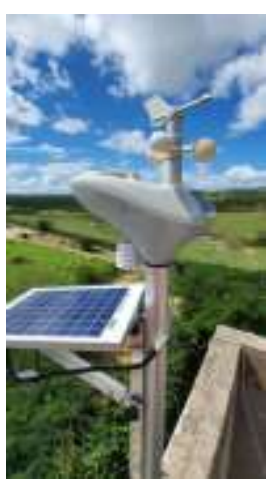

(b) Gravatá

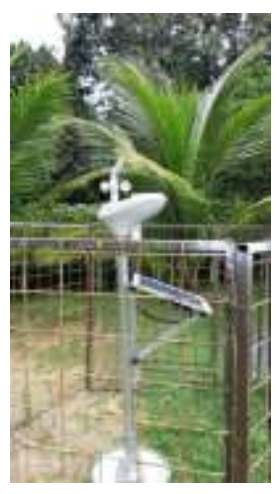

(c) APA Aldeia-Beberibe

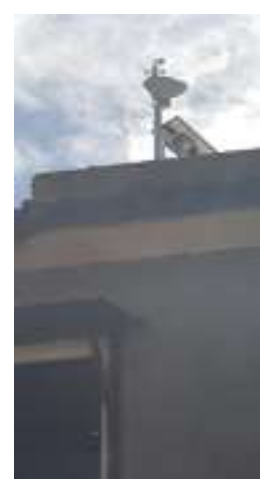

(d) Porto de Galinhas

Fonte: Autores.

A Tabela 1 ilustra uma medição instantânea dos dados meteorológicos dos 4 pontos de monitoramento, ocorrida no dia 10/07/2021 às 19:00h. Os dados da Tabela 1 demonstram que houve uma variabilidade interessante entre os pontos (vide linha "Maior diferença"), o que poderia nortear o processo decisório de um turista sobre locomoção, a depender de condições de temperatura, precipitação etc. Além disso, os dados coletados foram comparados em vários momentos distintos com dados fornecidos por serviços meteorológicos públicos existentes e em alguns casos, especialmente sobre velocidade e rajada de vento, há um erro nos serviços públicos de mais de $90 \%{ }^{4}$.

\footnotetext{
${ }^{4}$ Comparação entre a estação Taquaritinga do Norte e a informação fornecida pelo serviço Clima Tempo, coletado em 14/07/2021 às 14h
} 
Tabela 1 - Medição instantânea dos dados meteorológicos dos 4 pontos de monitoramento.

\begin{tabular}{lcccc}
\hline \multirow{2}{*}{ Estação } & \multicolumn{4}{c}{ Dados Meteorológicos } \\
\cline { 2 - 5 } & Temperatura $\left({ }^{\circ} \mathbf{C}\right)$ & Umidade (\%) & Precipitação (mm) & Vel. Vento (km//h) \\
\hline Taquaritinga do Norte & 20,9 & 87,0 & 0,6 & 5,4 \\
\hline Gravatá & 21,4 & 91,0 & 0,3 & 0,4 \\
\hline Porto de Galinhas & 27,7 & 81,0 & 0,3 & 23,4 \\
\hline APA Aldeia-Beberibe & 22,2 & 99,0 & 0,0 & 0,0 \\
\hline Maior diferença & $32,53 \%$ & $22,22 \%$ & $50 \%$ & $5845 \%$ \\
\hline
\end{tabular}

Fonte: Autores.

\subsection{Testes de usabilidade}

A solução proposta passou por um crivo de qualidade interno à equipe do projeto, seguindo uma metodologia típica para o desenvolvimento de software de pequeno/médio porte. Contudo, para não possuir apenas uma avaliação endógena por partes dos integrantes, foram conduzidos testes de usabilidade com um público-alvo que pudesse representar potenciais turistas e potenciais usuários da solução. Para realização dos testes de usabilidade foram aplicados formulários qualitativos e de satisfação, e foi executado um estudo de campo de caráter exploratório, avaliativo e moderado. Segundo (Nielsen, 1994) com apenas 5 participantes consegue-se insumos suficientes para encontrar cerca de $80 \%$ dos problemas de usabilidade, visando atingir um grau mais elevado de precisão, os testes executados neste projeto foram executados com 8 pessoas.

O estudo de campo foi realizado presencialmente com o intuito de capturar o momento da interação do usuário com o aplicativo e também foi realizada uma gravação do desempenho do participante durante os testes utilizando um aplicativo capaz de registrar a quantidade de cliques e o tempo gasto em cada tarefa. Isso permitiu coletar dados importantes sobre o comportamento do usuário, permitindo medir a eficácia e eficiência do aplicativo com bastante acurácia. $\mathrm{O}$ formulário aplicado durante os testes é composto por questões que visam avaliar critérios de facilidade, satisfação, visibilidade do status, compatibilidade entre o sistema e o mundo real, intuitividade. $\mathrm{O}$ formulário também abria espaço para que o participante opinasse, sugerisse ou relatasse incômodos e erros oriundos da experiência de uso do aplicativo.

As 5 (cinco) tarefas planejadas para os testes de usabilidade foram: a) alterar o idioma, realizar o cadastro de usuário e logar no aplicativo; b) acessar um ponto turístico, visualizar suas informações cli-máticas e retornar à tela home em seguida; c) acessar a câmera de vídeo de um ponto turístico e retornar à tela Home; d) acessar a área de FAQ, clicar sobre uma pergunta frequente e retornar à tela Home; e) sair do app. Cada tarefa possui uma sequência de passos planejados para o cenário ideal, que variam de 2 passos para a tarefa mais simples (tarefa "e") até 10 passos para a tarefa mais complexa (tarefa "a"). Por limitação de espaço, não serão reproduzidos os passos de todas as tarefas. Os resultados obtidos foram calculados em termos de métricas, compiladas por meio de fórmulas de classificadores. As métricas escolhidas para serem analisadas no teste de interação do usuário com o aplicativo foram basicamente tempo e quantidade de cliques. Quatro classificadores universais foram criados com o intuito de facilitar a compreensão dos resultados, sendo eles: Realização Ideal (RI), Realização Satisfatória $(R S)$, Realização Insa-tisfatória $(R I n)$ e Tarefa Incompleta (TI). Com relação ao Tempo, foi analisado o Tempo Encontrado $(T E)$ que é uma relação entre o Tempo Gasto $(T G)$ e o Tempo Necessário $(T N)$. Com relação à quantidade de cliques foi considerado o Número de Erros (NumErr), que é uma relação entre o Número de Cliques Total (NCT) e Cliques Necessários $(C N)$. O cálculo de TE é dado por: $T E=(T G-T N)$. O cálculo de NumErr é dado por NumErr $=N C T$-- $C N$. 
Foi desenvolvido um modelo próprio para análise dos testes de usabilidade com objetivo de facilitar a interpretação dos resultados coletados para cada usuário. Após análise dos vídeos oriundos do estudo de campo, os dados coletados foram armazenados para que ficassem or-ganizados de forma padronizada, evitando erros na fase posterior. A Figura 9 ilustra um dos resultados, representando o usuário US001. Neste quadro os campos em amarelo estão relacionados aos classificadores de Tempo e Cliques, os valores nele inseridos representam uma média do desempenho dos desenvolvedores do aplicativo para realização de cada tarefa individualmente; os campos em azul escuro servem para descrever o nome das tarefas; as células em azul claro serão preenchidas com os valores coletados do desempenho do usuário para Tempo e Cliques, enquanto as células verdes serão preenchidas com os classificadores $R I, R S, R I n$ e $T I$ conforme a interpretação dos resultados obtidos. Essas são todas as informações relacionadas a primeira etapa de desenvolvimento de testes.

Figura 9 - Ilustração do quadro de resultados de teste de usabilidade.

\begin{tabular}{|c|c|c|c|c|c|c|c|c|c|c|c|c|c|c|c|}
\hline COD. & \multicolumn{3}{|c|}{ TAREFA - A } & \multicolumn{3}{|c|}{ TAREFA - B } & \multicolumn{3}{|c|}{ TAREFA - C } & \multicolumn{3}{|c|}{ TAREFA - D } & \multicolumn{3}{|c|}{ TAREFA - E } \\
\hline \multirow[t]{4}{*}{ US001 } & $\mathrm{TN}$ & TG & $\mathrm{CL}$ & $\mathrm{TN}$ & TG & $\mathrm{CL}$ & $\mathrm{TN}$ & TG & $\mathrm{CL}$ & $\mathrm{TN}$ & TG & $\mathrm{CL}$ & TN & TG & $\mathrm{CL}$ \\
\hline & 65 & & & 15 & & & 30 & & & 22 & & & 05 & & \\
\hline & $\mathrm{CN}$ & NCT & $\mathrm{CL}$ & $\mathrm{CN}$ & NCT & $\mathrm{CL}$ & $\mathrm{CN}$ & NCT & $\mathrm{CL}$ & $\mathrm{CN}$ & NCT & $\mathrm{CL}$ & $\mathrm{CN}$ & NCT & $\mathrm{CL}$ \\
\hline & 10 & & & 03 & & & 05 & & & 03 & & & 02 & & \\
\hline
\end{tabular}

Fonte: Autores.

Além do estudo de campo, foi realizada uma pesquisa de satisfação baseada em um formulário próprio para descobrir o nível de dificuldade encontrado pelos usuários ao realizar as tarefas. No mesmo formulário, também foi testado o nível de similaridade dos ícones utilizados no sistema em relação ao mundo real, e por fim, coletou-se impressões pessoais do usuário quanto a beleza das telas, conforto visual em relação às cores utilizadas, problemas e sugestões a fim de aplicar melhorias futuras ao aplicativo. Com relação ao nível de dificuldade das tarefas, os usuários puderam classificar suas experiências em 4 níveis diferentes, a saber: muito fácil; fácil; difícil; e muito difícil. Doze perguntas com possibilidade de resposta de tex to curto mediram o nível interatividade dos ícones utilizados e testaram a heurística de compatibilidade entre o sistema e o mundo real, onde a imagem dos ícones foi apresentada aos usuários e uma pergunta com resposta aberta lhes indagavam a qual funcionalidade aquele ícone deveria corresponder no contexto do apli-cativo proposto. As respostas esperadas para cada imagem mostrada na ordem em que se encontram no formulário são: Pesquisar; Dados Pessoais; Tela Principal; Localização; Chat; Dúvidas; Vídeo; Cidade; Temperatura; Foto; Praia; Sair. Palavras idênticas ou que possuam o mesmo valor semântico que as elenca-das serão consideradas como corretas, em termos avaliativos. Com o intuito de testar a heurística de reconhecimento, as mesmas ima-gens foram mostradas, mas dessa vez acompanhadas de opções de múltiplas escolhas e ao todo 12 opções de respostas guiavam o usuário nas escolhas dos significados das imagens. Como parâmetro para analisar o sucesso desse questionamento, desejava-se encontrar um cenário com menos erros que o anterior, simulando um usuário com mais experiência, provando que com um pouco mais de uso, os ícones serviriam de modo a guiar os utilizadores facilmente através das telas do aplicativo proposto. A última seção de perguntas se iniciou buscando captar informações perti-nentes a satisfação do usuário. Uma pergunta sobre a beleza do aplicativo inicia a última seção, possuindo uma escala de 0-10 onde os usuários deixam suas notas e estas eram interpretadas da seguinte maneira: Muito Bonito: 10; Bonito: 9; Razoável: 8-7; Feio: 6-5; Muito Feio: 4-0. A segunda pergunta da última seção do formulário tenta descobrir a probabilidade de os usuários indicarem este aplicativo a amigos e conhecidos. Esta Pergunta é analisada aqui utilizando os conceitos de uma metodologia 
co-nhecida como Net Promoter Score (NPS) (Reichheld,2003). O NPS serve para medir o grau de satisfação dos clientes de variadas empresas atualmente, e consiste na classificação dos usuários em Promotores, Passivos/Neutros e Detratores. Destacase que esta mesma pergunta que realizada nesta seção se destacou das demais, por correlacionar 11 dos 14 casos estudados em artigo sobre satisfação e fidelização do usuário (Reichheld, 2003). As demais perguntas do formulário possuem possibilidade de resposta de texto longo para que os usuários tenham liberdade suficiente para falarem com riqueza de detalhes acerca de possíveis desconfortos visuais, erros, dificuldades ou sugestões de melhorias para futuras versões do aplicativo.

Os resultados obtidos a partir do teste de campo e da aplicação do formulário foram muito satisfatórios. Por limitação de espaço, serão reproduzidos apenas 2 gráficos relacionados com os testes de usabilidade. O gráfico da Figura 10a ilustra que 77,4\% dos usuários apresentaram resultado ideal ou satisfatório, 22,5\% apresentou resultado insuficiente, mas nenhum usuário deixou de fazer as tarefas solicitadas ( $0 \%$ para $T I$ ). O gráfico da Figura 10b ilustra que apenas $1 \%$ dos usuários consideraram o aplicativo difícil, enquanto que $99 \%$ consideraram muito fácil ou fácil. Além disso, foi obtido um valor de NPS $=75$, que é considerado excelente pela definição.

Figura 10 - Resultados obtidos por pesquisa de campo e formulário de satisfação.

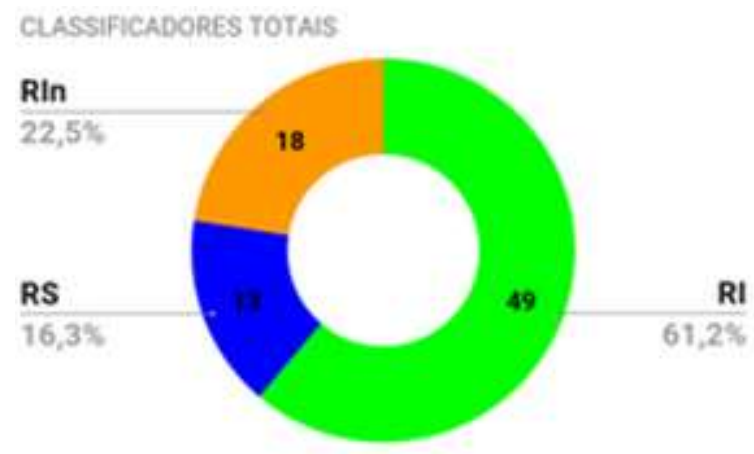

(a) Classificadores da pesquisa de campo.

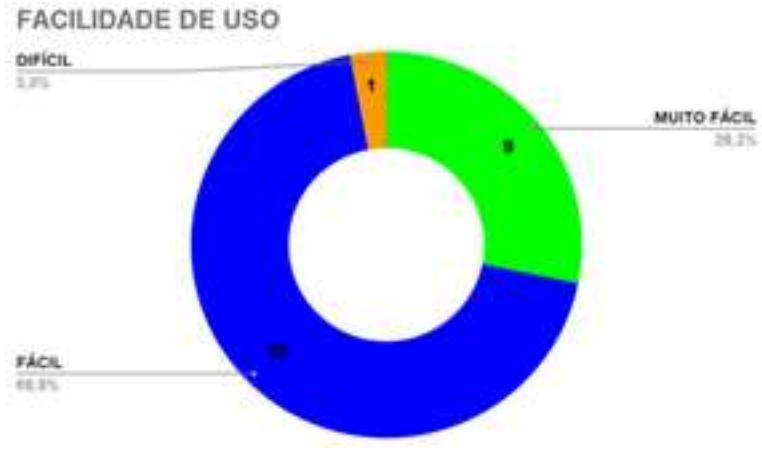

(b) Facilidade de uso, de acordo com formulário.

Fonte: Autores.

\subsection{Análise preliminar de desempenho}

A partir do momento em que se tem um protótipo funcional de um sistema modelado, surge a necessidade de avaliar o seu desempenho. Por tanto, para a produção de uma análise preliminar do sistema, adotou-se a técnica de avaliação por simulação (Jain, 1991), considerando teste de carga de usuários na plataforma Dojot. O teste de carga de usuários é importante para demonstrar o comportamento da plataforma de IoT, evidenciar a capacidade de atendimento de requisições simultâneas associadas ao desempenho do ambiente computacional em nuvem, além de servir de parâmetro norteador para ações de escalabilidade do projeto. Para a realização de testes de carga, adotou-se a ferramenta gratuita Locust, desenvolvida na linguagem Python, capaz de simular milhares de usuários reais realizando requisições ao sistema (Locust, 2020). Realizaramse testes com a ferramenta Locust, de maneira a simular 5.000 usuários em acessos simultâneos. Os parâmetros utilizados para realização do teste foram definidos pelo número total de usuários a serem simulados em 5.000, pela taxa de geração de novos usuários a cada 20 segundos e endereço da plataforma de IoT que recebe o teste de carga. A Tabela 2 apresenta os resultados de uma simulação, na qual obteve-se um total de 188.592 requisições ao final do teste, sendo que 75.429 requisições apresentaram falha, o equivalente a $40 \%$ do total de requisições. Contudo, salienta-se que as falhas obtidas no resultado da simulação ocorreram devido à negação de novas conexões à plataforma Dojot em detrimento à quantidade excessiva de solicitações simultâneas. Esse número pode ser melhorado com uma melhor infraestrutura do serviço de nuvem ou mesmo 
otimização da Dojot em futuras versões da plataforma. Observou-se que durante o ápice do teste de simulação de carga, o servidor Linux em nuvem atingiu $46,16 \%$ de utilização dos quatro núcleos virtuais de processamento ( $v C P U)$, demonstrando que o dimensionamento do servidor implementado em nuvem para o projeto é capaz de atender às altas demandas da aplicação, com sobra de recursos computacionais para utilizações e testes ainda mais agressivos.

Tabela 2 - Resultados de simulação de carga usando Locust.

\begin{tabular}{cccccccc}
\hline Indicador & Usuários & Requisições & Falhas & $\begin{array}{c}\text { Percentual } \\
\text { sucesso }\end{array}$ & $\begin{array}{c}\text { Percentual de } \\
\text { falha }\end{array}$ & $\begin{array}{c}\text { Taxa máxima de } \\
\text { geração de usuários }\end{array}$ & $\begin{array}{c}\text { Taxa máxima } \\
\text { de falha }\end{array}$ \\
\hline Valor & 5000 & 188592 & 75429 & $60 \%$ & $40 \%$ & 533 usuários/s & 246 falhas/s \\
\hline
\end{tabular}

Fonte: Autores.

\section{Considerações Finais}

Este trabalho apresentou uma proposta de solução tecnológica para o Turismo 4.0, com foco no nicho de turismo de natureza. A proposta consiste no uso de dispositivos IoT, computação em nuvem e aplicativo para dispositivos móveis, para permitir que os turistas possam avaliar as condições meteorológicas e visualizar vídeo em tempo real do ponto desejado, facilitando o processo decisório sobre locomoção e visitação dos pontos de interesse. Os protótipos das estações de monitoramento foram instalados em 4 (quatro) pontos turísticos do estado de Pernambuco e monitorados por um período de 3 meses por meio de um aplicativo para dispositivos móveis. O aplicativo foi desenvolvido para a plataforma Android e ficou disponível para que potenciais turistas pudessem fornecer sugestões sobre a solução. De acordo com as consultas realizadas com os usuários, o aplicativo apresentou-se intuitivo e as tarefas associadas com a solução foram executadas por todos os participantes. Além disso, $99 \%$ dos usuários classificam o aplicativo como de uso fácil ou muito fácil.

Futuras investigações podem considerar o uso de Inteligência Artificial para melhorar a experiência do usuário, modelando o aplicativo como um sistema de recomendação de pontos turísticos. Além disso, outros sensores podem ser acrescentados às estações de monitoramento futuramente, para coletar algumas categorias de dados que hoje não estão disponíveis.

\section{Agradecimentos}

Os autores agradecem o apoio financeiro fornecido pelo MCTI e UFRPE.

\section{Referências}

B2K. (2018). B2K Technology Solutions: Produtos. http://www.b2ktech.com.br/\#produtos.

Brasil. (2021). Governo Federal do Brasil. Ministério do Turismo. Relatório de Impacto da Pandemia de COVID-19 nos setores de turismo e cultura no Brasil. http://www.dadosefatos.turismo.gov.br/boletins/item/download/1175_000cfb701ec1f89133455ce5f0ed9caa.html.

Buhalis, D. (2019). Technology in tourism-from information communication technologies to eTourism and smart tourism towards ambient intelligence tourism: a perspective article. Tourism Review.

Caninos. (2018). Programa. https://caninosloucos.org/pt/program-pt.

Choudhari, S., Rasal, T., Suryawanshi, S., Mane, M., \& Yedge, S. (2017). Survey paper on internet of things: IoT. International Journal of Engineering Science and Computing, 7(4), 10564-10567.

CPqD. (2017). A plataforma dojot e os 10 princípios da "cidade como plataforma". https://www.cpqd.com.br/internet-das-coisas-iot-backup/a-plataformadojot-e-os-10-principios-da-cidade-como-plataforma. 
Research, Society and Development, v. 10, n. 9, e32210918271, 2021

(CC BY 4.0) | ISSN 2525-3409 | DOI: http://dx.doi.org/10.33448/rsd-v10i9.18271

Developer, A. (2009). Android Developer. línea]. https://developer. android. com.

Embarcados. (2019). Plataforma IoT - Como prototipar nossas ideias (Parte 1). https://www.embarcados.com.br/plataforma-iot-como-prototipar-nossasideias-parte-1.

Flauzino, M., Veríssimo, J., Terra, R., Cirilo, E., Durelli, V. H., \& Durelli, R. S. (2018). Are you still smelling it? A comparative study between Java and Kotlin language. In Proceedings of the VII Brazilian symposium on software components, architectures, and reuse (pp. 23-32).

Garcia, A., Linaza, M. T., Gutierrez, A., \& Garcia, E. (2019). Gamified mobile experiences: smart technologies for tourism destinations. Tourism Review.

Gcaba, O., \& Dlodlo, N. (2016). The internet of things for South African tourism. In 2016 IST-Africa Week Conference (pp. 1-8). IEEE.

Jain, R. (1990). The art of computer systems performance analysis: techniques for experimental design, measurement, simulation, and modeling. John Wiley \& Sons.

Khatri, I. (2019). Information technology in tourism \& hospitality industry: A review of ten years' publications. Journal of Tourism and Hospitality Education, 9, 74-87.

Locust. (2020). What is Locust? https://docs.locust.io/en/stable/what-is-locust.html.

Machado, L. P., \& Almeida, A. (2010). Inovação e Novas Tecnologias [recurso eletrônico]. Porto: SPI Sociedade Portuguesa de Informação.

Neves, C. S. B., de Souza, W. F. L., \& de Souza Carvalho, I. (2020). Tecnologia e espaços turísticos: aplicativos de turismo em áreas naturais no Brasil. Geografia: Publicações Avulsas, 2(1), 331-352.

NGINX. (2019). NGINX: o que é e por que utilizar? EVEO. https://www.eveo.com.br/blog/nginx/.

Nielsen, J. (1994). Usability engineering. Morgan Kaufmann.

Pasanen, K., Pesonen, J., Murphy, J., Heinonen, J., \& Mikkonen, J. (2019). Comparing Tablet and Virtual Reality Glasses for Watching Nature Tourism Videos. In Information and Communication Technologies in Tourism 2019 (pp. 120-131). Springer, Cham.

Pereira, A. S., Shitsuka, D. M., Parreira, F. J., \& Shitsuka, R. (2018). Metodologia da pesquisa científica Ed (pp. 3-9). UAB/NTE/UFSM: https://repositorio. ufsm. br/bitstream/handle/1/15824/Lic_Computacao_Metodologia-Pesquisa-Cientifica. pdf.

Santa Ana, A. G. (2019). Turismo brasileiro 4.0: do analógico ao digital. A digitalização e a mudança na venda de "pacotes" de viagens nas operadoras de turismo do Brasil (Dissertação).

Schulzrinne, H., Rao, A., \& Lanphier, R. (1998). RFC2326: Real time streaming protocol (RTSP).

Silva, F. (2013). Turismo na natureza como base do desenvolvimento turístico responsável nos Açores. Tese (Doutorado em geografia). Universidade de Lisboa.

SQLite. (2021). SQLLite Documentation. https://www.sqlite.org/docs.html.

Stankov, U., \& Gretzel, U. (2020). Tourism 4.0 technologies and tourist experiences: a human-centered design perspective. Information Technology \& Tourism, 22(3), 477-488.

UNWTO. (2019). International Tourism Highlights 2019 Edition. World Tourism Organization - UNWTO. http://www.dadosefatos.turismo.gov.br/images/demanda/International_Tourism_Highlights_2019_Edition.pdf. 